\title{
Comparison of Switch to Fingolimod or Interferon Beta/ Glatiramer Acetate in Active Multiple Sclerosis
}

\begin{abstract}
Anna He, MBBS; Tim Spelman, MBBS, MSc; Vilija Jokubaitis, PhD; Eva Havrdova, MD, PhD; Dana Horakova, MD, PhD; Maria Trojano, MD; Alessandra Lugaresi, MD; Guillermo Izquierdo, MD, PhD; Pierre Grammond, MD; Pierre Duquette, MD; Marc Girard, MD; Eugenio Pucci, MD, PhD; Gerardo Iuliano, MD; Raed Alroughani, MD; Celia Oreja-Guevara, MD; Ricardo Fernandez-Bolaños, MD; Francois Grand'Maison, MD; Patrizia Sola, MD; Daniele Spitaleri, MD; Franco Granella, MD; Murat Terzi, MD; Jeannette Lechner-Scott, MD; Vincent Van Pesch, MD, PhD; Raymond Hupperts, MD, PhD; José Luis Sánchez-Menoyo, MD; Suzanne Hodgkinson, MBBS, PhD; Csilla Rozsa, MD; Freek Verheul, MD; Helmut Butzkueven, MBBS, PhD; Tomas Kalincik, MD, PhD; for the MSBase Study Group
\end{abstract}

IMPORTANCE After multiple sclerosis (MS) relapse while a patient is receiving an injectable disease-modifying drug, many physicians advocate therapy switch, but the relative effectiveness of different switch decisions is often uncertain.

OBJECTIVE To compare the effect of the oral immunomodulator fingolimod with that of all injectable immunomodulators (interferons or glatiramer acetate) on relapse rate, disability, and treatment persistence in patients with active MS.

DESIGN, SETTING, AND PARTICIPANTS Matched retrospective analysis of data collected prospectively from MSBase, an international, observational cohort study. The MSBase cohort represents a population of patients with MS monitored at large MS centers. The analyzed data were collected between July 1996 and April 2014. Participants included patients with relapsing-remitting MS who were switching therapy to fingolimod or injectable immunomodulators up to 12 months after on-treatment clinical disease activity (relapse or progression of disability), matched on demographic and clinical variables. Median follow-up duration was 13.1 months (range, 3-80). Indication and attrition bias were controlled with propensity score matching and pairwise censoring, respectively. Head-to-head analyses of relapse and disability outcomes used paired, weighted, negative binomial models or frailty proportional hazards models adjusted for magnetic resonance imaging variables. Sensitivity analyses were conducted.

EXPOSURES Patients had received fingolimod, interferon beta, or glatiramer acetate for a minimum of 3 months following a switch of immunomodulatory therapy.

MAIN OUTCOMES AND MEASURES Annualized relapse rate and proportion of relapse-free patients, as well as the proportion of patients without sustained disability progression.

RESULTS Overall, 379 patients in the injectable group were matched to 148 patients in the fingolimod group. The fingolimod group had a lower mean annualized relapse rate $(0.31 \mathrm{vs}$ $0.42 ; 95 \% \mathrm{Cl}, 0.02-0.19 ; P=.009$ ), lower hazard of first on-treatment relapse (hazard ratio [HR], $0.74 ; 95 \% \mathrm{Cl}, 0.56-0.98 ; P=.04$ ), lower hazard of disability progression ( $\mathrm{HR}, 0.53$; $95 \% \mathrm{Cl}, 0.31-0.91 ; P=.02)$, higher rate of disability regression ( $\mathrm{HR}, 2.0 ; 95 \% \mathrm{Cl}, 1.2-3.3$; $P=.005)$, and lower hazard of treatment discontinuation (HR, 0.55; $P=.04)$ compared with the injectable group.

CONCLUSIONS AND RELEVANCE Switching from injectable immunomodulators to fingolimod is associated with fewer relapses, more favorable disability outcomes, and greater treatment persistence compared with switching to another injectable preparation following on-treatment activity of MS.

JAMA Neurol. 2015;72(4):405-413. doi:10.1001/jamaneurol.2014.4147

Published online February 9, 2015. Corrected on June 25, 2015.
Editorial page 387

Supplemental content at jamaneurology.com

CME Quiz at

jamanetworkcme.com
Author Affiliations: Author affiliations are listed at the end of this article.

Group Information: The list of MSBase Study Group coinvestigators and contributors is provided in the eAppendix in the Supplement.

Corresponding Author: Tomas Kalincik, MD, PhD, L4 Centre, Department of Neurology, Royal Melbourne Hospital, Grattan St, Parkville VIC 3050, Australia (tomas.kalincik@unimelb.edu.au). 
$\mathrm{T}$ he introduction of the disease-modifying drug fingolimod has had a substantial effect on the treatment of multiple sclerosis (MS). ${ }^{1-5}$ This novel oral agent is becoming widely used in highly active forms of MS, either as first-line therapy or after treatment failure while a patient is receiving traditional injectable disease-modifying therapies (ie, preparations of interferon beta and glatiramer acetate). ${ }^{6,7}$ Three landmark phase 3 clinical trials ${ }^{8-10}$ have demonstrated the efficacy of fingolimod in achieving more favorable clinical and radiologic end points compared with placebo (the FREEDOMS [FTY720 Research Evaluating Effects of Daily Oral Therapy in Multiple Sclerosis] and FREEDOMS II) or intramuscular interferon beta-1a (the TRANSFORMS [Trial Assessing Injectable Interferon vs FTY720 Oral in Relapsing-Remitting Multiple Sclerosis] trials). In the TRANSFORMS study, all patients experienced relapses before randomization, but approximately $45 \%$ of the participants were treatment naive. The active comparator to fingolimod in that study was $30 \mu \mathrm{g}$ of intramuscular interferon beta-1a, which had been shown to be inferior to $44 \mu \mathrm{g}$ of subcutaneous interferon beta-1 $\mathrm{a}^{11}$ or glatiramer acetate. ${ }^{12}$ Thus, the trials did not address in full the issue of the relative effectiveness of switching from interferon beta/glatiramer acetate to fingolimod vs switching between interferon beta/glatiramer acetate preparations in patients experiencing on-treatment relapses. We sought to address this issue using the MSBase registry data set. ${ }^{13}$

In the absence of randomized clinical trials, which are often costly and time consuming, the use of high-quality, protocol-driven observational data collections, such as MSBase, provides an important tool for comparisons of drug effectiveness in a real-world setting. Several validated statistical models have been successfully used to minimize the indication bias expected in observational data and thus allow meaningful interpretation of outcomes. ${ }^{14-16}$

In the MSBase data set, we used propensity score-based matching to create a quasi-randomized study design. ${ }^{17}$ We assessed the outcomes of matched patients whose treatment was switched to fingolimod or another interferon beta/glatiramer acetate preparation after experiencing breakthrough disease (ie, relapse, disability progression, or both) while receiving an interferon beta/glatiramer acetate preparation.

\section{Methods}

\section{Patient Selection and Data Acquisition}

The MSBase registry collects observational data for patients with MS as part of routine clinical care. The use of MSBase as a research platform was approved by the Melbourne Health Human Research Ethics Committee and by the local ethics committees in all participating centers (or exemptions were granted according to local laws and regulations). If required, written informed consent was obtained from enrolled patients. All patients recorded in the MSBase registry were screened using the following inclusion criteria: availability of the minimum data set; a recorded switch from interferon beta/glatiramer acetate (subcutaneous interferon beta-1a, $22 \mu \mathrm{g}$ or $44 \mu \mathrm{g}$ thrice weekly [Rebif], intramuscular interferon beta-1a, $30 \mu \mathrm{g}$ weekly [Avonex], subcutaneous interferon beta-1b, $250 \mu$ g every second day
[Betaferon/Betaseron], or subcutaneous glatiramer acetate, $20 \mathrm{mg} / \mathrm{d}$ [Copaxone]) to another interferon beta/glatiramer acetate preparation or oral fingolimod, $0.5 \mathrm{mg} / \mathrm{d}$ (Gilenya); at least 6 months of continuous interferon beta/glatiramer acetate treatment before the switch; a relapse and/or confirmed disability progression during interferon beta/glatiramer acetate treatment within the 12 months preceding the switch; a treatment gap of no longer than 3 months before beginning treatment with the switch medication; and at least 3 months of treatment persistence with the switch medication. Patients who previously received alemtuzumab, cladribine, infliximab, mitoxantrone hydrochloride, natalizumab, rituximab, dimethyl fumarate, teriflunomide, or fingolimod were excluded.

The minimum data set for each patient included date of birth, sex, MS center, disease course, dates of disease onset, clinic visits, relapses, dates of the beginning and end of treatment, and disability quantified with the Expanded Disability Status Scale $(\mathrm{EDSS})^{18}$ at baseline and at least 2 follow-up visits, at least 3 months apart, during switch therapy. When available, categorized evaluations of magnetic resonance imaging (within the year preceding baseline) and cerebrospinal fluid (at any time point) were reported by the treating neurologists.

All analyzed information was recorded as part of routine clinical practice between July 1996 and April 2014. The usual practice at most centers was real-time or near real-time data entry in association with clinical visits. The MSBase protocol stipulates minimum annual updates of the minimum data set, but patients with less-frequent updates were not excluded from the analysis if the above inclusion criteria were fulfilled. The data entry portal was either the iMed patient record system or the MSBase online data entry system. Quality assurance procedures were applied as described elsewhere. ${ }^{15}$

\section{Study End Points}

The primary outcomes of the study were the proportion of patients without relapses and the annualized relapse rate (ARR); secondary outcomes were time to progression or regression of disability confirmed at 3 months and time to treatment discontinuation.

A relapse was defined as the occurrence of new symptoms or exacerbation of existing symptoms persisting for at least 24 hours in the absence of concurrent illness or fever and occurring at least 30 days after a previous relapse. Individual ARRs were calculated as the annualized number of recorded relapses between baseline and a censoring event.

Disability was scored by accredited scorers using the EDSS (online Neurostatus certification ${ }^{18}$ was required at each center), excluding any EDSS score recorded within 30 days of a previous relapse. Progression, according to the EDSS, was defined as an increase of 1 or more EDSS steps ( $\geq 1.5$ EDSS steps if the baseline EDSS score was 0) sustained for 3 or more months. Regression, according to the EDSS, was defined as a decrease of 1 or more EDSS steps (1.5 EDSS steps if the baseline EDSS score was 1.5) sustained for 3 or more months.

\section{Matching}

Propensity score matching was used to match patients from each treatment arm by baseline clinical and demographic vari- 
ables; the method and its validity have been described elsewhere. ${ }^{15}$ The propensity of switching to either interferon beta/glatiramer acetate or fingolimod was estimated for all included patients using a multivariable logistic regression model (MatchIt package for R). ${ }^{19}$ The following variables at the time of the treatment switch were used for matching purposes: age, country, duration of MS, total number of previous treatment starts, the EDSS score at baseline, the number of relapses in the preceding 6 and 12 months, the type of recent disease activity preceding the treatment switch (relapse, disability progression, or both), and the type of interferon beta/glatiramer acetate preparation used before the switch. Variables associated with a switch to fingolimod at a significance level of $P<.10$ were used to generate individual propensity scores for each patient. Patients in the fingolimod and interferon beta/ glatiramer acetate groups were matched in a variable ratio of up to $1: 6$, using nearest-neighbor matching without replacement and a caliper of 0.3 SDs of the propensity score. Closeness of the match was evaluated using cumulative and mean propensity distances between groups as described elsewhere. ${ }^{15}$

\section{Statistical Analysis}

The statistical analysis was performed using R, version 3.0.2. ${ }^{20}$ Categorical and continuous variables at baseline were compared between the groups with $\chi^{2}$ and unpaired $t$ tests in the unmatched cohort and with weighted McNemar and weighted, paired $t$ tests in the matched cohort. To account for potential systematic differences in treatment persistence and the follow-up period, pairwise censoring of on-study follow-up was applied (with the exception of the treatment persistence analysis), that is, the follow-up period for each matched patient pair was determined by the shorter of the 2 patient follow-up periods.

Differences in time to first relapse, time to confirmed disability progression, and time to confirmed disability regression between groups were assessed using weighted, frailty-adjusted, multivariable Cox proportional hazards regression models (with the frailty representing a random effect adjusting for the matched probability of treatment assignation within pairs) adjusted for baseline T2 lesion number (trichotomized according to the McDonald diagnostic criteria ${ }^{21}$ into 1-8 lesions, $\geq 9$ lesions, or unavailable). The risk of discontinuation was evaluated with a weighted, frailty-adjusted Cox model adjusted for the categorized baseline T2 lesion number. The proportionality of hazards was assessed with the Schoenfeld global test. ${ }^{22}$ Differences in the ARR were assessed using weighted negative binomial regression with a cluster effect for matched patient pairs and adjusted for the trichotomized baseline T2 lesion number. To evaluate the sensitivity of this analysis to differential reporting of the low-severity relapses, an analysis including only the relapses treated with corticosteroids was conducted.

Sensitivity of the analysis to the type of postswitch interferon beta/glatiramer acetate preparation was tested by comparing the switch to fingolimod with the switch to subcutaneous interferon beta-1a, $22 \mu \mathrm{g}$ or $44 \mu \mathrm{g}$ thrice weekly, and switch to any of the injectable preparations, excluding $30 \mathrm{mg}$ of intramuscular interferon beta-1a. The sensitivity of the analysis of relapse outcomes to the inclusion criteria was evaluated by repeating the analyses among patients included re- gardless of their preswitch disease activity and with no required follow-up EDSS scores; however, the baseline EDSS score was required. In this sensitivity analysis, a very narrow caliper of 0.01 was used to control indication bias. Two sensitivity analyses were performed to validate the results among patients with at least 12 and 18 months of postswitch follow-up. Another sensitivity analysis excluded patients switching between the preparations of interferon beta. To evaluate the robustness of the analysis in relation to nonrecognized confounders of treatment assignation, the Rosenbaum sensitivity test of the Hodges-Lehmann $\Gamma$ was conducted for the analyses of ARR. ${ }^{23}$

\section{Results}

\section{Primary Analysis}

A total of 790 patients met the inclusion criteria (Table 1 and eTable 1 in the Supplement) and were included in the calculation of propensity score. Although preswitch treatment with intramuscular interferon beta-1a was more common among patients switching to another interferon beta/glatiramer acetate preparation, use of subcutaneous interferon beta-1a was more common among patients switching to fingolimod (eTable 2 in the Supplement). The following variables were associated with the switch to fingolimod: higher baseline EDSS score (odds ratio [OR], 1.17; 95\% CI, 1.01-1.33; $P=.05$ ), recent relapse (OR, 3.44; 95\% CI, 2.65-4.23; $P=.002)$, recent relapse and disability progression (OR, 3.38; 95\% CI, 2.55-4.21; $P=.004$ ), and higher number of previous treatment starts (OR, 2.55; 95\% CI, 2.19-2.91; $P<$.001). Preswitch treatment with intramuscular interferon beta-1b was associated with a switch to another interferon beta/glatiramer acetate preparation (OR, 5.7; 95\% CI, 5.03-6.37; $P<$.001). Country of residence was associated with treatment allocation (ORs, 5.5-43.1; $P \leq .006$ ).

The propensity score-matching procedure resulted in 148 patients in the fingolimod group matched to 379 patients in the interferon beta/glatiramer acetate group (Figure 1; distribution of propensity scores is available in eFigure 1 in the Supplement). The matching procedure significantly decreased the mean difference in propensity scores between the groups ( 0.40 prematch to 0.04 postmatch per patient) and improved the match on all of the considered variables (Table 1). Only the country of treatment remained different between groups after matching. Uncensored follow-up duration after the switch to fingolimod was shorter than that after the switch to interferon beta/glatiramer acetate (median, 14.9 vs 32.8 months). Median pairwise-censored follow-up time was 13.1 months (range, 3-80 months).

Fingolimod was associated with a decreased hazard of first on-treatment relapse compared with interferon beta/ glatiramer acetate (hazard ratio [HR], 0.74; 95\% CI, 0.56-0.98; $P=.04$ ) (Figure 2). The mean (SD) ARR values during switch therapy were $0.31(0.59)$ and 0.42 (0.68) in the fingolimod and interferon beta/glatiramer acetate groups, respectively ( $P=.009$ ), with a between-group difference of 0.10 relapses per year (95\% CI, 0.02-0.19).

Fingolimod was associated with a decreased hazard of confirmed disability progression compared with interferon beta/ 


\begin{tabular}{|c|c|c|c|c|c|c|c|c|}
\hline \multirow[b]{2}{*}{ Characteristic } & \multicolumn{4}{|c|}{ Before Matching } & \multicolumn{4}{|c|}{ After Matching } \\
\hline & $\begin{array}{l}\text { Interferon Beta/ } \\
\text { Glatiramer } \\
\text { Acetate }\end{array}$ & Fingolimod & Cohen $d$ & $\begin{array}{c}P \\
\text { Value }\end{array}$ & $\begin{array}{l}\text { Interferon Beta/ } \\
\text { Glatiramer } \\
\text { Acetate }\end{array}$ & Fingolimod & Cohen $d$ & $\begin{array}{c}P \\
\text { Value }\end{array}$ \\
\hline Patients, No. & 562 & 228 & & & 379 & 148 & & \\
\hline Female sex, №. (\%) & 410 (72.9) & 155 (67.9) & & .22 & $270(71.2)$ & $103(69.6)$ & & .74 \\
\hline Age, median (IQR), y & $36(29-42)$ & $38(32-44)$ & 0.22 & .005 & $36(30-42)$ & $37(31-44)$ & 0.14 & .78 \\
\hline \multicolumn{9}{|l|}{ Country, No. (\%) } \\
\hline Australia & $12(2.1)$ & $51(22.4)$ & & $<.001$ & $31(8.2)$ & $18(12.2)$ & & .07 \\
\hline Canada & $72(13.8)$ & $15(6.6)$ & & .01 & $38(10.0)$ & $14(9.5)$ & & .90 \\
\hline Czech Republic & $136(24.1)$ & $41(18.0)$ & & .06 & $86(22.7)$ & $30(20.0)$ & & .50 \\
\hline Spain & $58(10.3)$ & $49(21.5)$ & & $<.001$ & $93(24.5)$ & $32(21.6)$ & & .44 \\
\hline Italy & $227(40.3)$ & $41(18.0)$ & & $<.001$ & $106(28.0)$ & $38(25.7)$ & & .51 \\
\hline The Netherlands & $15(3.6)$ & $6(2.6)$ & & $>.99$ & $11(2.9)$ & $4(2.7)$ & & .92 \\
\hline Elsewhere & $42(7.5)$ & $25(10.9)$ & & .10 & $15(4.0)$ & $12(8.1)$ & & .03 \\
\hline Disease duration, median (IQR), y & $6.8(3.5-11.4)$ & $7.5(4.0-13.4)$ & 0.22 & .002 & $7.3(4.0-12.1)$ & $7.2(3.7-12.8)$ & 0.08 & .80 \\
\hline \multicolumn{9}{|l|}{ Treatment starts, No. (\%) } \\
\hline 1 & $483(85.9)$ & $150(65.8)$ & & $<.001$ & $307(81.0)$ & $106(71.6)$ & & .86 \\
\hline 2 & 67 (11.9) & $57(25.0)$ & & $<.001$ & $61(16.1)$ & $32(21.6)$ & & .32 \\
\hline 3 & $11(1.9)$ & $19(8.3)$ & & $<.001$ & $10(2.6)$ & $9(6.1)$ & & .11 \\
\hline$>3$ & $1(<0.1)$ & $2(<0.1)$ & & .23 & $1(<0.1)$ & $1(<0.1)$ & & $>.99$ \\
\hline \multicolumn{9}{|l|}{ Baseline therapy, №. (\%) } \\
\hline Interferon beta-1a, $30 \mu \mathrm{g} \mathrm{IM}$ & $319(56.7)$ & $40(17.6)$ & & $<.001$ & $90(23.7)$ & $32(21.6)$ & & .51 \\
\hline Interferon beta- $1 \mathrm{~b}, 250 \mu \mathrm{g} \mathrm{SC}$ & $70(12.5)$ & $51(22.4)$ & & $<.001$ & $80(21.1)$ & $32(21.6)$ & & .88 \\
\hline Interferon beta-1a, $22 \mu \mathrm{g} / 44 \mu \mathrm{g} \mathrm{SC}$ & $106(18.9)$ & $85(37.2)$ & & $<.001$ & $137(36.9)$ & $55(37.2)$ & & .84 \\
\hline Glatiramer acetate, $20 \mathrm{mg} \mathrm{SC}$ & 67 (11.9) & $52(23.8)$ & & .001 & $72(19.0)$ & $29(19.6)$ & & .90 \\
\hline Baseline disability (EDSS score), median (IQR) & $2.5(1.5-3.5)$ & $2.5(1.5-4.0)$ & 0.12 & .14 & $2.5(1.5-4.0)$ & $2.5(2.0-4.0)$ & 0.05 & .42 \\
\hline \multicolumn{9}{|l|}{ Prebaseline disease activity, №. (\%) } \\
\hline Disability progression & $107(19.0)$ & $24(10.5)$ & & .004 & $43(11.3)$ & $15(10.1)$ & & .69 \\
\hline Relapse & $251(44.7)$ & $122(53.5)$ & & .02 & $191(50.4)$ & $74(50.0)$ & & $>.99$ \\
\hline Both & $204(36.3)$ & $82(36.0)$ & & .91 & $145(38.3)$ & 59 (39.9) & & .72 \\
\hline Relapse count: 6 mo before baseline, mean (SD) & $0.7(0.6)$ & $0.7(0.6)$ & 0.11 & .19 & $0.7(0.6)$ & $0.7(0.6)$ & 0.05 & .90 \\
\hline Relapse count: 12 mo before baseline, mean (SD) & $1.2(0.9)$ & $1.2(0.7)$ & 0.04 & .64 & $1.2(0.8)$ & $1.3(0.7)$ & 0.02 & .47 \\
\hline \multicolumn{9}{|l|}{ MRI: hyperintense T2 lesions, №. (\%) } \\
\hline Missing & $356(63.3)$ & $136(59.6)$ & & \multirow{3}{*}{$<.001$} & $246(64.9)$ & $86(58.1)$ & & \multirow{3}{*}{$<.001$} \\
\hline $1-8$ & $138(24.6)$ & $7(3.1)$ & & & $84(22.2)$ & $4(2.7)$ & & \\
\hline$\geq 9$ & $68(12.1)$ & $85(37.3)$ & & & $49(12.9)$ & $58(39.2)$ & & \\
\hline \multicolumn{9}{|l|}{ MRI: contrast-enhancing lesions, No. (\%) } \\
\hline Missing & $373(66.4)$ & $166(72.8)$ & & \multirow{3}{*}{.001} & $260(68.6)$ & $106(71.6)$ & & \multirow{3}{*}{.006} \\
\hline 0 & $165(29.4)$ & $40(17.5)$ & & & $104(27.4)$ & $23(15.5)$ & & \\
\hline$\geq 1$ & $24(4.3)$ & $22(9.6)$ & & & $15(4.0)$ & $19(12.8)$ & & \\
\hline \multicolumn{9}{|l|}{ Cerebrospinal fluid, No. (\%) } \\
\hline Missing & $304(54.1)$ & $149(65.4)$ & & \multirow{3}{*}{.70} & $211(55.7)$ & $90(60.8)$ & & \multirow{3}{*}{$>.99$} \\
\hline Abnormal & $222(39.5)$ & $66(28.9)$ & & & $148(39.1)$ & $51(34.5)$ & & \\
\hline Normal & $36(6.4)$ & $13(5.7)$ & & & $20(5.3)$ & $7(4.7)$ & & \\
\hline
\end{tabular}

Abbreviations: EDSS, Expanded Disability Status Scale, IM, intramuscular; $\mathrm{IQR}$, interquartile range; $\mathrm{MRI}$, magnetic resonance image; $\mathrm{SC}$, subcutaneous.

a Unmatched continuous variables were compared using a Cohen $d$ and 2-tailed, unpaired $t$ test; unmatched categorical variables were compared using a $\chi^{2}$

test. Matched continuous variables were compared using a paired, weighted $t$ test; matched categorical variables were compared using a weighted, paired McNemar test. glatiramer acetate (HR, 0.53; 95\% CI, 0.31-0.91; $P=.02)$ (Figure 3A). In addition, fingolimod was associated with an increased probability of disability regression compared with interferon beta/glatiramer acetate (HR, 2.0; 95\% CI, 1.2-3.3; $P=$.005) (Figure 3B).
Treatment persistence during the follow-up period was higher in the fingolimod group compared with the interferon beta/glatiramer acetate group. The rate of treatment discontinuation at 24 months was $17.5 \%$ and $26.8 \%$, respectively (HR, 0.55; 95\% CI, 0.31-0.98; $P$ = .04) (eFigure 2 in the Supplement). 
Sensitivity Analyses

Based on the Rosenbaum sensitivity test, the HodgesLehmann $\Gamma$ for the primary analysis of ARR was 1.2, suggesting that a hidden confounder of a magnitude of more than $20 \%$ of the estimated propensity score would be required to change the present statistical inference.

Of all recorded relapses, $63.3 \%$ were treated with corticosteroids. When the analysis of relapse outcomes was repeated with only the corticosteroid-treated relapses, the result of the primary analysis was replicated (mean [SD] ARR, 0.23 [0.51] with fingolimod and 0.27 [0.53] with interferon beta/ glatiramer acetate; $P=.02$ ).

Further sensitivity analyses were conducted by repeating the analysis in a matched cohort of patients switching therapy to fingolimod or subcutaneous interferon beta-1a or to any injectable agent except intramuscular interferon beta-1a (Table 2). These findings have replicated the outcomes of the primary analysis with the exception of treatment persistence. In addition, the analysis of the sensitivity of the relapse outcomes to preswitch activity confirmed the primary analysis among patients included regardless of their preswitch MS activity and the availability of postswitch EDSS time points. The 2 analyses that included only patients with longer postswitch follow-up periods confirmed most of the results of the primary analysis but showed only statistically nonsignificant trends for the comparisons of ARR and, in the case of analysis of patients with follow-up of 18 months or longer, of confirmed disability progression events. Finally, the sensitivity analysis excluding patients switching between the preparations of interferon beta confirmed the relatively lower postswitch relapse activity among patients switching to fingolimod but showed only nonsignificant trends for both disability outcomes.

\section{Discussion}

In this retrospective analysis of prospectively acquired observational data from MSBase, we found that switching to fingolimod resulted in improved relapse outcomes (ARR and proportion of patients without relapses) compared with switching to interferon beta/glatiramer acetate in patients previously experiencing breakthrough disease activity while receiving interferon beta/ glatiramer acetate. Switching to fingolimod was associated with more favorable disability outcomes compared with the switch to interferon beta/glatiramer acetate, reducing the risk of 3-month confirmed disability progression by $47 \%$ and doubling the likelihood of disability regression during treatment.

The TRANSFORMS phase 3 randomized clinical trial ${ }^{10} \mathrm{com}-$ paring fingolimod with intramuscular interferon beta-1a demonstrated superior efficacy of fingolimod on relapse activity, number of gadolinium-enhancing lesions on magnetic resonance imaging, and total lesion burden. In the trial's 2-year extension and a subgroup analysis in the context of previously active disease despite immunomodulatory therapy, patients in the interferon beta-1a group who switched treatment to fingolimod also experienced a reduction in ARR. ${ }^{24,25}$ Three previous studies have compared fingolimod with other preparations of interferon beta/glatiramer acetate: a retrospective study $^{26}$ of the number of claims for intravenous corticosteroid treatment during a 540-day period in a US patient cohort receiving fingolimod or interferon beta/glatiramer acetate and 
Figure 2. Proportions of Patients Without Relapses in the Matched Cohorts

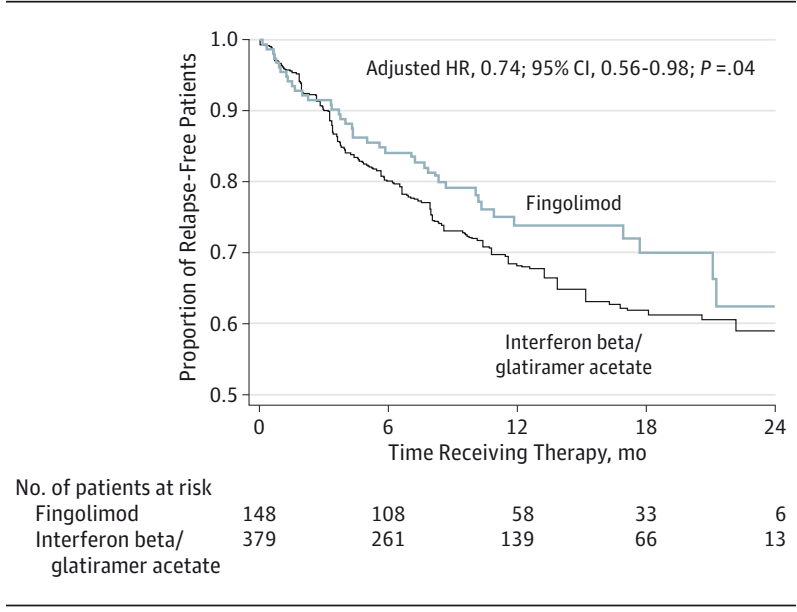

HR indicates hazard ratio.

2 network meta-analyse ${ }^{27,28}$ of randomized clinical trials that indirectly compared the efficacy of fingolimod with that of each of the interferon beta/glatiramer acetate preparations. These studies demonstrated relative reduction in relapse activity among patients receiving fingolimod after adjusting for baseline demographic and clinical differences (52\% reduction in the number of corticosteroid claims, 30\%-48\% reduction in ARR, and $18 \%-23 \%$ increase in the proportion of relapse-free patients at 1 year of treatment initiation). To our knowledge, the present study is the first to demonstrate improved shortterm disability outcomes in a population of patients with active treated MS. With respect to the 3-month confirmed disability outcomes used in our analysis, this observation most likely reflects improved prevention of neurologic disability accumulated as a result of MS relapses with incomplete recovery. ${ }^{29,30}$ In a similar manner, higher probability of reduced disability suggests clinical stabilization of disease in patients with previously active MS (included in the study on the basis of recent on-treatment relapse and/or progression of disability).

Although the primary analysis in our study showed higher treatment persistence following switching therapy to fingolimod compared with interferon beta/glatiramer acetate, this observation was not replicated by any of the sensitivity analyses and therefore should be interpreted with caution considering the potential preparation- or matching-specific effects. In contrast, 3 sensitivity analyses replicated in full the relapse and disability outcomes observed in the primary analysis. The 3 sensitivity analyses using extended minimum postswitch follow-up durations and eliminating switching between the interferon preparations showed nonsignificant trends for several outcomes, including the ARR and some of the disability outcomes. The nonsignificance was most likely determined by the loss of power owing to the relatively small subcohort size. Thus, the sensitivity analyses demonstrated that the observed clinical outcomes were largely independent of the definitions of preswitch MS activity, variables used in the
Figure 3. Confirmed Disability Status at 3 Months in the Matched Cohorts

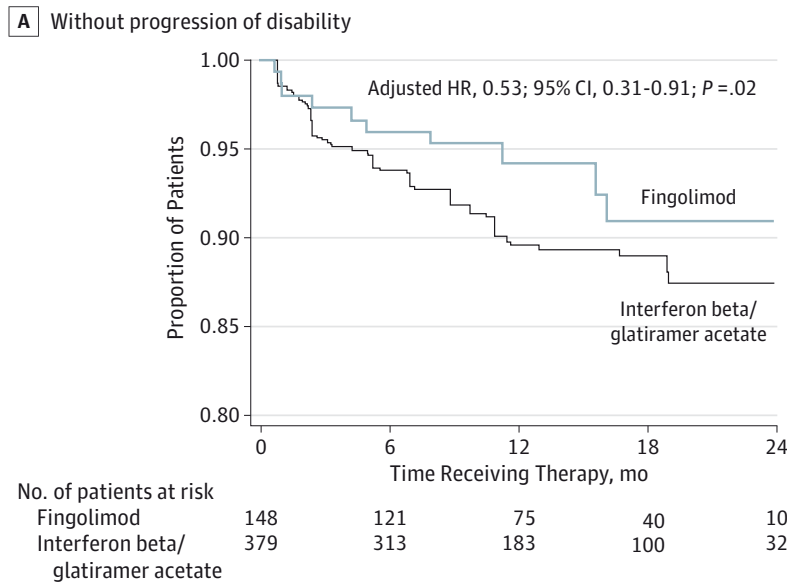

B With regression of disability

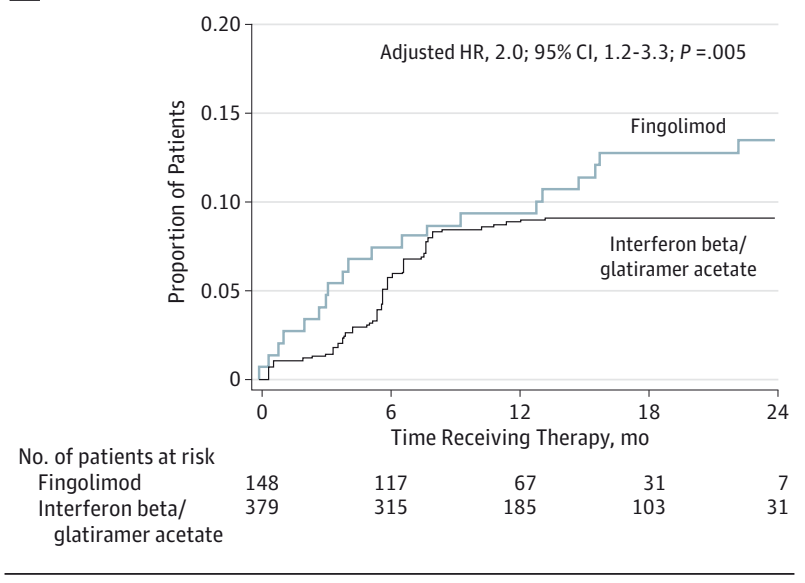

A, Patients without progression. B, Patients with regression. HR indicates hazard ratio.

matching procedure, and the known, albeit small, differences between the injectable immunomodulators. ${ }^{31,32}$

Although the expected indication bias was eliminated or markedly reduced by matching the compared cohorts on the determinants of treatment allocation, the propensity scorebased matching did not adjust for unknown confounders. We observed that our analysis may be vulnerable to a hypothetical unidentified confounder constituting more than $20 \%$ of the SD of all identified determinants of treatment allocation if its distribution was asymmetrical. In addition, the lack of blinding in the present study may have introduced detection and reporting bias; however, one may argue that the follow-up protocols for fingolimod and interferon beta/glatiramer acetate are largely comparable and that the magnitude of such a bias would therefore be minimal. Even though the usual data entry practice at MSBase is near-real-time entry, some variables, such as relapse-related information, may be susceptible to recall bias. Moreover, most relapses were not confirmed by a documented change in the EDSS score. It is therefore reassuring that the ARR reported in MSBase is consistent with the ARRs reported in contemporary clinical trials. ${ }^{33}$ In addition, the 


\begin{tabular}{|c|c|c|c|}
\hline Outcome & Fingolimod & $\begin{array}{l}\text { Interferon Beta/ } \\
\text { Glatiramer } \\
\text { Acetate }\end{array}$ & $P$ Value \\
\hline \multicolumn{4}{|l|}{ Switch to Fingolimod vs SC Interferon Beta-1a } \\
\hline Matched patients, No. & 71 & 168 & \\
\hline On-treatment relapse, OR $(95 \% \mathrm{Cl})$ & $0.51(0.33-0.78)$ & & .002 \\
\hline Annualized relapse rate, mean (SD) & $0.29(0.60)$ & $0.48(0.76)$ & .003 \\
\hline \multicolumn{4}{|l|}{ Disability, OR $(95 \% \mathrm{Cl})$} \\
\hline Progression & $0.29(0.12-0.72)$ & & .007 \\
\hline Regression & $2.1(1.0-4.2)$ & & .04 \\
\hline Treatment persistence, OR $(95 \% \mathrm{Cl})$ & $0.63(0.30-1.33)$ & & .23 \\
\hline Hodges-Lehmann $\Gamma$ & 1.2 & & \\
\hline \multicolumn{4}{|c|}{ Switch to Fingolimod vs Injectable Therapies Except IM Interferon Beta-1a } \\
\hline Matched patients, No. & 160 & 428 & \\
\hline On-treatment relapse, OR (95\% Cl) & $0.53(0.41-0.70)$ & & $<.001$ \\
\hline Annualized relapse rate, mean (SD) & $0.30(0.57)$ & $0.45(0.66)$ & $<.001$ \\
\hline \multicolumn{4}{|l|}{ Disability, OR $(95 \% \mathrm{Cl})$} \\
\hline Progression & $0.39(0.22-0.70)$ & & .001 \\
\hline Regression & $2.0(1.3-3.1)$ & & .002 \\
\hline Treatment persistence, OR $(95 \% \mathrm{Cl})$ & $0.68(0.40-1.18)$ & & .15 \\
\hline Hodges-Lehmann $\Gamma$ & 1.2 & & \\
\hline \multicolumn{4}{|c|}{ Patients Regardless of Preswitch MS Activity and No Required EDSS Follow-up } \\
\hline Matched patients, No. & 340 & 876 & \\
\hline On-treatment relapse, OR $(95 \% \mathrm{Cl})$ & $0.61(0.51-0.75)$ & & $<.001$ \\
\hline Annualized relapse rate, mean (SD) & $0.23(0.51)$ & $0.43(0.72)$ & $<.001$ \\
\hline Treatment persistence, OR $(95 \% \mathrm{Cl})$ & $0.86(0.63-1.17)$ & & .33 \\
\hline Hodges-Lehmann $\Gamma$ & 1.0 & & \\
\hline \multicolumn{4}{|l|}{ Patients With Postswitch Follow-up at $\geq 12 \mathrm{mo}$} \\
\hline Matched patients, No. & 295 & 93 & \\
\hline Pairwise-censored follow-up, median (IQR), mo & $19(15-22)$ & & \\
\hline On-treatment relapse, OR $(95 \% \mathrm{Cl})$ & $0.68(0.51-0.91)$ & & .01 \\
\hline Annualized relapse rate, mean (SD) & $0.32(0.58)$ & $0.37(0.52)$ & .10 \\
\hline \multicolumn{4}{|l|}{ Disability, OR $(95 \% \mathrm{Cl})$} \\
\hline Progression & $0.51(0.31-0.84)$ & & .008 \\
\hline Regression & $2.7(1.7-4.5)$ & & .001 \\
\hline Treatment persistence, OR $(95 \% \mathrm{Cl})$ & $0.73(0.41-1.31)$ & & .29 \\
\hline \multicolumn{4}{|l|}{ Patients With Postswitch Follow-up $\geq 18$ mo } \\
\hline Matched patients, No. & 187 & 53 & \\
\hline Pairwise-censored follow-up, median (IQR), mo & $21(19-25)$ & & \\
\hline On-treatment relapse, OR (95\% Cl) & $0.56(0.39-0.81)$ & & .002 \\
\hline Annualized relapse rate, mean (SD) & $0.29(0.52)$ & $0.35(0.48)$ & .10 \\
\hline \multicolumn{4}{|l|}{ Disability, OR $(95 \% \mathrm{Cl})$} \\
\hline Progression & $0.89(0.48-1.62)$ & & .69 \\
\hline Regression & $2.5(1.4-3.9)$ & & $<.001$ \\
\hline Treatment persistence, OR $(95 \% \mathrm{Cl})$ & $0.89(0.40-1.96)$ & & .76 \\
\hline \multicolumn{4}{|c|}{ Switch to Fingolimod vs Switch Between Interferons and Glatiramer Acetate } \\
\hline Matched patients, No. & 199 & 119 & \\
\hline On-treatment relapse, OR $(95 \% \mathrm{Cl})$ & $0.58(0.39-0.86)$ & & .006 \\
\hline Annualized relapse rate, mean (SD) & $0.28(0.59)$ & $0.39(0.64)$ & .01 \\
\hline \multicolumn{4}{|l|}{ Disability, OR $(95 \% \mathrm{Cl})$} \\
\hline Progression & $0.55(0.26-1.20)$ & & .13 \\
\hline Regression & $1.1(0.6-2.2)$ & & .71 \\
\hline Treatment persistence, OR $(95 \% \mathrm{Cl})$ & $0.80(0.44-1.45)$ & & .46 \\
\hline Hodges-Lehmann $\Gamma$ & 1.2 & & \\
\hline
\end{tabular}

Abbreviations: EDSS, Expanded Disability Status Scale; IM, intramuscular; IQR, interquartile range; $\mathrm{OR}$, odds ratio; SC, subcutaneous. 
detection rate of relapses in the present study was comparatively higher than that reported in the phase 2 and 3 clinical trials for fingolimod; it is therefore unlikely that the present study was affected by underreporting of relapses. Discontinuation rates reported in our study were determined by a combination of treatment efficacy, tolerability, and convenience of treatment administration or follow-up. The inclusion criteria eliminated patients who discontinued treatment within the initial 3 months after the switch, which may have biased the persistence analysis if differential discontinuation between the groups occurred within this time. Given that the information concerning reasons for stopping therapy was not routinely recorded, we were unable to examine the trends within on-study treatment discontinuation.
Although the analyses of observational data do not serve as a substitute for trial data, our study provides real-world evidence, representative of clinical practice in tertiary MS centers, to support clinical decision making that is highly relevant to the management of active MS.

\section{Conclusions}

Using real-world MS outcomes data from patients with active MS despite treatment with injectableimmunomodulatory agents, we have shown that switching therapy to oral fingolimod is more effective in controlling relapse activity and accumulation of disability than switching to another injectable disease-modifying drug.

\section{ARTICLE INFORMATION}

Accepted for Publication: November 10, 2014.

Published Online: February 9, 2015.

doi:10.1001/jamaneurol.2014.4147.

Author Affiliations: Department of Neurology, Royal Melbourne Hospital, Melbourne, Australia (He, Butzkueven, Kalincik); Department of Medicine, University of Melbourne, Melbourne, Australia (Spelman, Jokubaitis, Butzkueven, Kalincik); Department of Neurology and Center of Clinical Neuroscience, General University Hospital, Prague, Czech Republic (Havrdova, Horakova); Department of Neurology and Center for Clinical Neuroscience, Charles University, Prague, Czech Republic (Havrdova, Horakova); Department of Basic Medical Sciences, Neuroscience, and Sense Organs, University of Bari, Bari, Italy (Trojano); MS Center, Department of Neuroscience, Imaging, and Clinical Sciences, University G. d'Annunzio, Chienti, Italy (Lugaresi); Department of Neurology, Hospital Universitario Virgen Macarena, Sevilla, Spain (Izquierdo); Department of Neurology, Hotel-Dieu de Lévis, Lévis, Quebec, Canada (Grammond); Department of Neurology, Hôpital Notre Dame, Montreal, Quebec, Canada (Duquette, Girard); Neurology Unit, Azienda Sanitaria Unica Regionale Marche, Macerata, Italy (Pucci); Department of Neurology, Ospedali Riuniti di Salerno, Salerno, Italy (Iuliano); Department of Neurology, Amiri Hospital, Kuwait City, Kuwait (Alroughani); Multiple Sclerosis Unit, University Hospital San Carlos, Madrid, Spain (Oreja-Guevara); Department of Neurology, Hospital Universitario Virgen de Valme, Seville, Spain (Fernandez-Bolaños); Neuro Rive-Sud, Hôpital Charles LeMoyne, Quebec City, Quebec, Canada (Grand'Maison); Department of Neurology, Nuovo Ospedale Civile San Agostino, Modena, Italy (Sola); Department of Neurology, Azienda Ospedaliera di Rilievo Nazionale, San Giuseppe Moscati, Avellino, Italy (Spitaleri); Institute of Neurology, University of Parma, Parma, Italy (Granella); Medical Faculty, Department of Neurology, Ondokuz Mayis University, Samsun, Turkey (Terzi); Department of Medicine, John Hunter Hospital, Newcastle, Australia (Lechner -Scott); Hunter Medical Research Institute, University of Newcastle, Newcastle, Australia (Lechner-Scott); Department of Neurology, Cliniques Universitaires Saint-Luc, Brussels, Belgium (Van Pesch); Department of Neurology, Orbis Medical Center, Sittard, the Netherlands (Hupperts); Department of Neurology, Hospital de Galdakao-Usansolo, Galdakao, Spain (Sánchez
-Menoyo); Department of Nephrology, Liverpool Hospital, Liverpool, Australia (Hodgkinson); Department of Neurology, Liverpool Hospital, Liverpool, Australia (Hodgkinson); Department of Neurology, Jahn Ferenc Teaching Hospital, Budapest, Hungary (Rozsa); Neurology Unit, Groen Hart Ziekenhuis, Gouda, the Netherlands (Verheul); Department of Neurology, Box Hill Hospital, Monash University, Melbourne, Australia (Butzkueven).

Author Contributions: Drs He, Spelman, Butzkueven, and Kalincik contributed equally to the manuscript. Dr Kalincik had full access to all the data in the study and takes responsibility for the integrity of the data and the accuracy of the data analysis.

Study concept and design: He, Spelman, Butzkueven, Kalincik.

Acquisition, analysis, or interpretation of data: All authors.

Drafting of the manuscript: He, Spelman, Kalincik. Critical revision of the manuscript for important intellectual content: Spelman, Jokubaitis, Havrdova, Horakova, Trojano, Lugaresi, Izquierdo, Grammond, Duquette, Girard, Pucci, Iuliano, Alroughani, Oreja -Guevara, Fernandez-Bolaños, Grand'Maison, Sola, Spitaleri, Granella, Terzi, Lechner-Scott, Van Pesch, Hupperts, Sánchez-Menoyo, Hodgkinson, Rozsa, Verheul, Butzkueven, Kalincik.

Statistical analysis: He, Spelman, Kalincik.

Obtained funding: Butzkueven, Kalincik. Administrative, technical, or material support: Jokubaitis, Duquette, Oreja-Guevara, Terzi, Van Pesch, Hupperts, Rozsa, Verheul, Butzkueven, Kalincik.

Study supervision: Havrdova, Trojano, Lugaresi,

Pucci, Alroughani, Fernandez-Bolaños,

Grand'Maison, Granella, Lechner-Scott

Butzkueven, Kalincik.

Conflict of Interest Disclosures: Dr Spelman received compensation for travel from Biogen Idec. Dr Jokubaitis has received conference travel support from Novartis. Dr Havrdova received speaker honoraria and consultant fees from Biogen Idec, Genzyme, Merck Serono, Novartis, and Teva, as well as support for research activities from Biogen Idec and Merck Serono. Dr Horakova received speaker honoraria and consulting fees from Biogen Idec, Merck Serono, Novartis, and Teva, as well as support for research activities from Biogen Idec. Dr Trojano received speaking honoraria from Bayer Schering, Biogen Idec, Merck Serono, Novartis, Sanofi, and Teva, as well as research grants from Biogen Idec, Merck Serono, and Novartis. Dr Lugaresi is a Bayer Schering, Biogen Idec, Genzyme, and Merck Serono advisory board member; she has received travel grants and honoraria from Bayer Schering, Biogen Idec, Merck Serono, Novartis, Sanofi, and Teva; research grants from Bayer Schering, Biogen Idec, Merck Serono, Novartis, Sanofi, and Teva; and travel and research grants from the Associazione Italiana Sclerosi Multipla; and is a Consultant of Fondazione Cesare Serono. Dr Izquierdo received speaking honoraria from Biogen Idec, Merck Serono, Novartis, Sanofi, and Teva. Dr Grammond is a Biogen Idec, Novartis, and Teva-Neuroscience advisory board member; a consultant for Merck Serono; received payments for lectures from the Canadian Multiple Sclerosis Society, Merck Serono, and Teva-Neuroscience; and received grants for travel from Novartis and Teva -Neuroscience. Dr Girard received consulting fees from Biogen Idec, Novartis, Sanofi-Genzyme, and Teva Canada Innovation; lecture payments from EMD Serono, Novartis, and Teva Canada Innovation; as well as a research grant from Canadian Institutes of Health Research. Dr Pucci served on scientific advisory boards for Biogen Idec and Genzyme; he has received honoraria and travel grants from Bayer Schering, Biogen Idec, Genzyme, Lundbeck, Merck Serono, Novartis, Sanofi, Teva, and UCB, as well as received travel grants from Associazione Marchigiana Sclerosi Multipla e altre malattie neurologiche. Dr luliano had travel /accommodations/meeting expenses funded by Bayer Schering, Biogen Idec, Merck Serono, Novartis, Sanofi, and Teva. Dr Alroughani received honoraria from Bayer, Biologix, GSK, Merck Sorono, and Novartis and served on advisory boards for Biologix, Merck Sorono, and Novartis. Dr Oreja -Guevara received honoraria as a consultant on scientific advisory boards from Bayer Schering, Biogen Idec, Merck Serono, Novartis, and Teva and has participated in clinical trials/other research projects by Biogen Idec, GSK, Novartis, and Teva. Dr Grand'Maison received honoraria from Biogen Idec, Genzyme, Novartis, and Roche. Dr Spitaleri received honoraria as a consultant on scientific advisory boards from Bayer Schering, Novartis, and Sanofi as well as compensation for travel from Biogen Idec, Merck Serono, Novartis, Sanofi, and Teva. Dr Terzi received travel grants from Bayer Schering, Merck Serono, Novartis, and Teva and has participated in clinical trials by Novartis, Roche, and Sanofi. Dr Lechner-Scott has accepted travel compensation from Biogen, Merck Serono, and Novartis; her institution receives the honoraria for 
talks and advisory board commitment and clinic support from Bayer Health Care, Biogen Idec, CSL, Merck Serono, Novartis, and Sanofi-Genzyme. Dr Van Pesch has served on advisory boards for Biogen Idec, Novartis Pharma, and Sanofi-Genzyme; has received travel grants and consultancy fees from Bayer Schering, Biogen Idec, Merck Serono, Novartis Pharma, Sanofi, and Sanofi-Genzyme; and has received research grants from Bayer Schering. Dr Hupperts received honoraria as a consultant on scientific advisory boards from Biogen Idec, Merck Serono, SanofiGenzyme, and Teva; research funding from Biogen Idec and Merck Serono; and speaker honoraria from Sanofi-Genzyme. Dr Rozsa has received speaker honoraria from Bayer Schering, Biogen Idec, and Novartis and conference and travel expense compensation from Bayer Schering, Biogen Idec, Merck Serono, and Teva. Dr Verheul is an advisory board member for Biogen, Merck Serono, Novartis, and Teva. Dr Butzkueven has served on scientific advisory boards for Biogen Idec, Novartis, and Sanofi and has received conference travel support from Biogen Idec, Novartis, and Sanofi; he serves on steering committees for trials conducted by Biogen Idec and Novartis and has received research support from Biogen Idec, Merck Serono, and Novartis.

Dr Kalincik received compensation for travel and consultancy honoraria from BioCSL, Biogen Idec, Genzyme, Merck Serono, Novartis, Sanofi, and Teva. No other disclosures were reported.

Funding/Support: This investigator-initiated analysis was financially supported by Novartis Pharma, Multiple Sclerosis Research Australia (fellowship 11-054), National Health and Medical Research Council (fellowships 628856 and 1071124 , grant 1032484, and Centre For Research Excellence grant 1001216), and MSBase Foundation (a not-for -profit organization that receives support from Bayer Schering, Biogen Idec, Merck Serono, Novartis Pharma, and Sanofi).

\section{Role of the Funder/Sponsor: The funding} organizations had no role in the design and conduct of the study; collection, management, analysis, and interpretation of the data; preparation, review, or approval of the manuscript; and decision to submit the manuscript for publication.

Group Information: The list of MSBase Study Group coinvestigators and contributors is given in the eAppendix in the Supplement.

Correction: This article was corrected on June 25 , 2015 , to fix the point of origin for the graph's curves in Figure 3B.

\section{REFERENCES}

1. Brinkmann V, Davis MD, Heise CE, et al. The immune modulator FTY72O targets sphingosine 1-phosphate receptors. J Biol Chem. 2002;277(24):21453-21457.

2. Kappos L, Antel J, Comi G, et al; FTY720 D2201 Study Group. Oral fingolimod (FTY720) for relapsing multiple sclerosis. N Engl J Med. 2006; 355(11):1124-1140.

3. O'Connor P, Comi G, Montalban X, et al; FTY720 D2201 Study Group. Oral fingolimod (FTY720) in multiple sclerosis. Neurology. 2009;72(1):73-79.
4. Montalban X, Comi G, O'Connor P, et al. Oral fingolimod (FTY720) in relapsing multiple sclerosis. Mult Scler. 2011;17(11):1341-1350.

5. Comi G, O'Connor P, Montalban X, et al; FTY720D2201 Study Group. Phase II study of oral fingolimod (FTY720) in multiple sclerosis: 3-year results. Mult Scler. 2010;16(2):197-207.

6. Fazekas F, Bajenaru O, Berger T, et al. How does fingolimod (Gilenya ${ }^{\circledR}$ ) fit in the treatment algorithm for highly active relapsing-remitting multiple sclerosis? Front Neurol. 2013;4:10.

7. Fazekas F, Berger T, Fabjan TH, et al. Fingolimod in the treatment algorithm of relapsing remitting multiple sclerosis: a statement of the Central and East European (CEE) MS Expert Group. Wien Med Wochenschr. 2012;162(15-16):354-366.

8. Calabresi PA, Radue EW, Goodin D, et al. Safety and efficacy of fingolimod in patients with relapsing-remitting multiple sclerosis (FREEDOMS II): a double-blind, randomised, placebo-controlled, phase 3 trial. Lancet Neurol. 2014;13(6):545-556.

9. Kappos L, Radue EW, O'Connor P, et al; FREEDOMS Study Group. A placebo-controlled trial of oral fingolimod in relapsing multiple sclerosis. N Engl J Med. 2010;362(5):387-401.

10. Cohen JA, Barkhof F, Comi G, et al; TRANSFORMS Study Group. Oral fingolimod or intramuscular interferon for relapsing multiple sclerosis. N Engl J Med. 2010;362(5):402-415.

11. Panitch H, Goodin DS, Francis G, et al; EVIDENCE Study Group; Evidence of Interferon Dose-Response: European North American Comparative Efficacy; University of British Columbia MS/MRI Research Group. Randomized, comparative study of interferon beta-1a treatment regimens in MS: the EVIDENCE Trial. Neurology. 2002;59(10):1496-1506

12. Lublin FD, Cofield SS, Cutter GR, et al; CombiRx Investigators. Randomized study combining interferon and glatiramer acetate in multiple sclerosis. Ann Neurol. 2013;73(3):327-340.

13. Butzkueven $\mathrm{H}$, Chapman J, Cristiano $\mathrm{E}$, et al. MSBase: an international, online registry and platform for collaborative outcomes research in multiple sclerosis. Mult Scler. 2006;12(6):769-774.

14. Trojano M, Pellegrini F, Paolicelli D, Fuiani A, Di Renzo V. observational studies: propensity score analysis of non-randomized data. Int MS J. 2009; 16(3):90-97.

15. Kalincik T, Spelman T, Trojano M, et al; MSBase Study Group. Persistence on therapy and propensity matched outcome comparison of two subcutaneous interferon beta 1a dosages for multiple sclerosis. PLoS One. 2013;8(5):e63480. doi:10.1371/journal.pone.0063480.

16. Goodin DS, Jones J, Li D, et al; 16 -Year Long-Term Follow-up Study Investigators. Establishing long-term efficacy in chronic disease. PLoS One. 2011;6(11):e22444. doi:10.1371/journal .pone.0022444.

17. Rosenbaum PR, Rubin DB. Reducing bias in observational studies using subclassification on the propensity score. J Am Stat Assoc. 1984;79(387): 516-524.
18. Kurtzke JF. Rating neurologic impairment in multiple sclerosis: an expanded disability status scale (EDSS). Neurology. 1983;33(11):1444-1452.

19. Ho DS, Imai K, King G, Stuart EA. Matchlt: nonparametric preprocessing for parametric causal inference. J Stat Softw. 2011;42(8):1-28.

20. R Development Core Team. R: A Language and Environment for Statistical Computing. Vienna, Austria: R Foundation for Statistical Computing; 2011.

21. Polman $\mathrm{CH}$, Reingold SC, Banwell $\mathrm{B}$, et al. Diagnostic criteria for multiple sclerosis. Ann Neurol. 2011;69(2):292-302.

22. Schoenfeld D. Chi-squared goodness-of-fit tests for the proportional hazards regression model. Biometrika. 1980;67(1):145-153.

23. Rosenbaum PR. Observational Studies. 2nd ed. New York, NY: Springer-Verlag; 2002.

24. Khatri B, Barkhof F, Comi G, et al; TRANSFORMS Study Group. Comparison of fingolimod with interferon beta-la in relapsing-remitting multiple sclerosis. Lancet Neurol. 2011;10(6):520-529.

25. Cohen JA, Barkhof F, Comi G, et al. Fingolimod versus intramuscular interferon in patient subgroups from TRANSFORMS. J Neurol. 2013;260 (8):2023-2032.

26. Bergvall N, Makin C, Lahoz R, et al. Relapse rates in patients with multiple sclerosis switching from interferon to fingolimod or glatiramer acetate. PLoS One. 2014;9(2):e88472.

27. Roskell NS, Zimovetz EA, Rycroft CE, Eckert BJ, Tyas DA. Annualized relapse rate of first-line treatments for multiple sclerosis. Curr Med Res Opin. 2012;28(5):767-780.

28. Del Santo F, Maratea D, Fadda V, Trippoli S, Messori A. Treatments for relapsing-remitting multiple sclerosis: summarising current information by network meta-analysis. Eur J Clin Pharmacol. 2012;68(4):441-448.

29. Lublin FD, Baier M, Cutter G. Effect of relapses on development of residual deficit in multiple sclerosis. Neurology. 2003;61(11):1528-1532. 30. Hirst C, Ingram G, Pearson O, Pickersgill T, Scolding N, Robertson N. Contribution of relapses to disability in multiple sclerosis. J Neurol. 2008; 255(2):280-287.

31. Filippini G, Del Giovane C, Vacchi L, et al. Immunomodulators and immunosuppressants for multiple sclerosis. Cochrane Database Syst Rev. 2013;6:CD008933. doi:10.1002/14651858 .CD008933.pub2.

32. Kalincik T, Jokubaitis V, Izquierdo G, et al; MSBase Study Group. Comparative effectiveness of glatiramer acetate and interferon beta formulations in relapsing-remitting multiple sclerosis [published online December 5, 2014]. Mult Scler.

33. Kalincik T, Vivek V, Jokubaitis V, et al; MSBase Study Group. Sex as a determinant of relapse incidence and progressive course of multiple sclerosis. Brain. 2013;136(pt 12):3609-3617. 